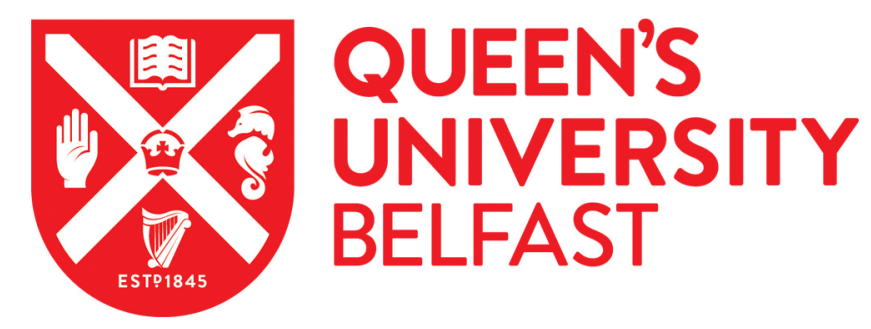

\title{
Measurement of Deep Tissue Implanted Antenna Efficiency Using a Reverberation Chamber
}

\author{
El-Saboni, Y., Magill, M. K., Conway, G. A., Cotton, S., \& Scanlon, W. G. (2017). Measurement of Deep Tissue \\ Implanted Antenna Efficiency Using a Reverberation Chamber. IEEE Journal of Electromagnetics, RF and \\ Microwaves in Medicine and Biology, 1(2), 90-97. https://doi.org/10.1109/JERM.2017.2765638 \\ Published in: \\ IEEE Journal of Electromagnetics, RF and Microwaves in Medicine and Biology
}

Document Version:

Peer reviewed version

Queen's University Belfast - Research Portal:

Link to publication record in Queen's University Belfast Research Portal

Publisher rights

@ 2017 IEEE. This work is made available online in accordance with the publisher's policies. Please refer to any applicable terms of use of the publisher.

\section{General rights}

Copyright for the publications made accessible via the Queen's University Belfast Research Portal is retained by the author(s) and / or other copyright owners and it is a condition of accessing these publications that users recognise and abide by the legal requirements associated with these rights.

Take down policy

The Research Portal is Queen's institutional repository that provides access to Queen's research output. Every effort has been made to ensure that content in the Research Portal does not infringe any person's rights, or applicable UK laws. If you discover content in the Research Portal that you believe breaches copyright or violates any law, please contact openaccess@qub.ac.uk. 


\title{
Measurement of Deep Tissue Implanted Antenna Efficiency Using a Reverberation Chamber
}

\author{
Yomna El-Saboni, Student Member, IEEE, Matthew K. Magill, Student Member, IEEE, Gareth A. Conway, \\ Member, IEEE, Simon L. Cotton, Senior Member, IEEE and William G. Scanlon, Senior Member, IEEE
}

\begin{abstract}
We investigate the use of a reverberation chamber for the experimental measurement of deep implant antenna radiation efficiency. The technique was able to measure the extremely low efficiencies associated with deep implant antennas inside a muscle tissue-mimicking liquid phantom. Results were obtained for a range of insulated and un-insulated antennas with efficiencies as low as $0.06 \%$. Analysis showed that while measurement errors were dominated by positioning variability, spurious feed cable radiation is still a significant factor that must be considered. Depending on the radiation characteristics of the antenna under test and the feed cable routing within the phantom, cable radiation could lead to errors of up to $4.5 \mathrm{~dB}$.
\end{abstract}

\section{Index Terms}

Radiation efficiency, implant antenna, tissue equivalent phantom, reverberation chamber, cable effects.

\section{INTRODUCTION}

$\mathbf{R}$ ECENT innovations in Implantable Medical Devices (IMDs) are implemented in a range of clinical applications including monitoring of disease progression in chronically ill patients [1], pacemakers, cochlear implants and nerve stimulators for neurological conditions [2]. However, one of the main challenges with IMDs is to efficiently communicate with external off-body (bed-side, or in-home) and on-body wearable devices. This would facilitate applications that require continuous real-time feedback such as an insulin pump based on blood-glucose level monitoring or a smart prosthetic limb [3]. To develop the wireless communications needed, the frequency of operation, bandwidth, power source and other essential factors must be optimized and chosen accordingly [4]. For RF based communications, the IMD antenna is a critical element with the main tradeoffs being physical size, frequency of operation and radiation characteristics [5]. In the past, the 402-405 MHz Medical Implant Communications Service (MICS) band was considered due to lower body tissue attenuation [6]. However, lower frequencies limit the available bandwidth and antenna miniaturization. Accordingly, recent work considered operation at higher frequencies including the Industrial, Scientific, and Medical band at 2400-2483.5 MHz and the MedRadio Band at $2360-2400 \mathrm{MHz}$ allocated by the U.S. Federal Communications Commission [7], [8].

Given the nature of implant applications, their physical size is a critical issue [9]. However, physically small antennas are extermely challenging, not only in terms of design and manufacture but also in testing and performance evaluation. Additionally, the lossy tissue environment within the body and its non-homogeneous structural complexity make it more challenging to accurately characterize implantable antennas [5]. In particular measuring radiation efficiency of deep tissue implants is difficult because the high attenuation rate of body tissues results in extremely low efficiency values. Therefore, it is no surprise that in the literature most empirical implant antenna performance characterization studies have focused on measuring reflection coefficient and bandwidth, and single or dual plane far-field radiation pattern. Notably, significantly less attention has been directed towards the measurement of radiation efficiency [10]. Limited work in this area includes [11] where simulations were performed to estimate radiation efficiency for different implantable antennas. An early study looked at the radiation efficiency of implantable antennas using numerical simulation and the results showed good agreement with representative in-vivo measurements [12]. Despite its importance, radiation efficiency is rarely measured and most studies utilize simulations and limited validation of reflection coefficient in a tissue-equivalent phantom.

Therefore, to address this need, and for the first time in literature, a reverberation chamber (RC) has been shown to effectively measure deep implant antenna efficiency. Previously, the RC technique has been shown to accurately measure the radiation efficiency for electrically small and wearable antennas [13]-[15]. We now extend this to include implant antennas with extremely low efficiencies below $0.1 \%$. Another important contribution is that we recognize and characterize the main limiting factor of this approach which is feed cable radiation. Furthermore, to ensure the generality and applicability of our results, we conduct our experiments using a range of antennas with differing radiation characteristics.

\section{Measuring Implant Antenna Efficiency}

Empirical measurement of radiation efficiency can be extremely challenging especially with low gain antennas. Common approaches include measuring the directivity and the gain to indirectly estimate the efficiency [16], or Wheeler Cap techniques [13], [17] where two measurements are made; one in free space and one in a shielded cavity to suppress radiation [16]. Recently, the use of the RC method has gained popularity as it is convenient and accurate [18]. An RC provides a statistical environment which consists of a conductive structure with complex shaped metallic mode stirring paddles which are used to continuously change the boundary conditions of the 
fields within the chamber [13]. While there are no previous studies of RC-based deep tissue implant antenna efficiency measurements, the technique was briefly mentioned in relation to a sub-surface (shallow) implant [19] where the antenna was positioned only $2.5 \mathrm{~mm}$ below the body surface in skin tissue. This antenna is not implanted it is only tissue loaded since the wave penetration depth at $2.48 \mathrm{GHz}$ within skin is $22 \mathrm{~mm}$. Furthermore, in [19] the measured efficiency was around $40 \%$ which is much higher than that expected for deep tissue implanted antennas. As shown in the sequel, we are able to demonstrate the accurate measurement of IMD antennas with efficiency lower than $1 \%$.

\section{A. Tissue Equivalent Phantoms}

Tissue-equivalent phantoms are designed with permittivity and conductivity characteristics that closely mimic human tissues. They facilitate the accurate evaluation of implant antenna radiation characteristics as well as providing repeatability and circumventing the ethical and practical difficulties associated with testing in humans. Tissue-equivalent phantoms are categorised as either numerical or experimental [20]. Standardized numerical phantoms have many benefits since they provide an accurate representation of the properties of human tissues and can be readily distributed. Simulations can also be used to provide detailed modeling of the implant antenna and yield a better understanding of the signal propagation and interaction with body tissues and structures. Nonetheless, while simulations can be informative and electromagnetically accurate, they only illustrate the ideal situation according to the specified model and assumed parameters. Therefore, phantom specifications are highly critical, considering that the human body is a highly dispersive environment with different tissue shapes and frequency dependent properties [21]. For practical reasons, physical phantoms are usually more simplified than their numerical equivalents. However, they still provide a reproducible and consistent environment for empirical testing and when combined with numerical simulations are extremely powerful in terms of validation and verification of implant antenna performance. Therefore, we utilized a liquid tissueequivalent phantom for our experimental work and verified the results through numerical simulations.

\section{B. Feed Cable Effects}

When characterizing the performance of IMD antennas there are many factors which can perturb the measurement results. In particular, most experimental setups involve the use of a feed cable to connect instrumentation to the antenna under test (AUT) within the physical phantom. This can introduce additional errors particularly since the AUT feed point impedance can be affected by the introduction of the feed cable [22] and, since perfectly balanced conditions are difficult to establish with a miniaturized antenna, currents may flow on the outside of the feed cable leading to spurious radiation [23]. While feed cable effects will not be a concern for a clinically deployed medical implant, they are an important consideration for laboratory testing. To ascertain the potential impact of feed cable effects upon implant antenna performance, [21] considered the case when an implant antenna operating in the MICS band was placed in an empty phantom. The authors then repeated their experiments using a filled tissue equivalent liquid phantom where the cable was in direct contact with the solution. By studying the antenna reflection coefficient for four different feed scenarios the results showed that the cable can have a considerable impact. Hence, to further contribute to understanding in this area, we consider the impact of the feeding cable on the radiation efficiency measurements.

To overcome the limitations associated with coaxial cables, others suggested using alternatives such as optical fiber. In [23], an optical fiber feed and fiber optic to RF transducer were used in the characterization of a monopole antenna. In particular, the noise level, dynamic range and frequency response were measured and compared to results obtained using a coaxial cable. The authors demonstrated a clear discrepancy between the results obtained using both techniques. While using a fiber optic based approach may appear advantageous, the relatively large size of the transducer $(20 \times 23.5 \times 10 \mathrm{~mm}$ [23]) is problematic for implanted antenna characterization. Alternatively, a more expensive Opto-Electric Field Sensor can be used [24] but its physical size still remains an issue.

As described by [13], [25], radiation efficiency, $\eta$, of an antenna is a key performance metric which can be defined as the ratio of the radiated power $P_{r}$ and the input power accepted by the antenna $P_{i}$. For implant antennas the radiated power is typically determined external to the body or the tissue equivalent phantom which surrounds the implant. $P_{i}$ can be defined as the summation of $P_{r}$ and the losses within both the body tissues and the antenna structure itself, $P_{\text {loss }}$. In the measurement of implant antennas, $P_{r}$ consists of the power radiated by the AUT $\left(P_{\text {aut }}\right)$ and any spurious radiation from the feed cable $\left(P_{\text {cable }}\right)$ [26], [27]. Therefore, the measured implant antenna efficiency, $\eta_{\text {meas }}$, is:

$$
\eta_{\text {meas }}=\frac{P_{r}}{P_{\text {loss }}+P_{r}}=\frac{P_{\text {aut }}+P_{\text {cable }}}{P_{\text {loss }}+P_{\text {aut }}+P_{\text {cable }}}
$$

It is understood that $P_{\text {cable }}$ depends on the measurement set up. In particular, feed cable radiation could have both dampened (fields reduced by the proximity of the lossy phantom materials) and un-dampened components depending on its positioning within the phantom. The minimization of cable radiation is usually not a significant problem in cases where the antenna is an efficient radiator and these effects can be discounted. However, with implant antennas the relative effect of cable radiation is greater due to the extremely low radiation efficiencies involved. This is particularly relevant in deep tissue applications, defined here as use-cases in which the implanted antenna is surrounded by a tissue thickness of more than one penetration depth. As a result, power losses can be expected to be greater than $\frac{1}{e^{2}}(\eta<13.5 \%)$. Hence, we can consider the implications of this if we define the estimated (measured) radiation efficiency as:

$$
\eta_{\text {meas }}=\eta \cdot \frac{P_{r}}{P_{\text {aut }}}=\eta \cdot \frac{P_{\text {aut }}+P_{\text {cable }}}{P_{\text {aut }}}
$$

As can be deduced from (2), $\eta_{\text {meas }}$ will only approach the true radiation efficiency when the power radiated by the cable is minimized. 


\section{EXPERIMENTAL METHODOLOGY}

The use of repeated trials, different antenna radiation characteristics and the variation of feed cable arrangements allowed the investigation of the validity of the RC technique for measuring deep implant antenna efficiency whilst also providing insight into both the sensitivity and limitations of the approach. The technique used in this study involves the use of a known efficiency discone reference antenna [28], as discussed thoroughly in [13]. The efficiency results were obtained for the $2.2-2.3 \mathrm{GHz}$ frequency range as this was optimal for matching all of the AUTs and it is also adjacent to the spectrum space allocated for MedRadio. It is worth highlighting that the experimental technique presented here can be applied to MICS or any other relevant frequency band depending on the specification of the RC.

The RC used in the experiments was described in [15] and is based on a $2.4 \times 2.4 \times 2.4 \mathrm{~m}$ shielded room with an offset internal metallic wall to make it cuboidal and additional fixed diagonal metallic baffles. Spatial stirring is achieved with a rotational table and two mechanical plate stirrers. The RC was operated using control and data acquisition software provided by Bluetest $\mathrm{AB}$. For polarization stirring, the $\mathrm{RC}$ has wideband monopole test antennas $(400-3000 \mathrm{MHz})$ on the roof and on two walls of the chamber. Measurements utilized a Rohde and Schwarz ZVB-8 vector network analyzer over the $100 \mathrm{MHz}$ bandwidth of interest $(2.2-2.3 \mathrm{GHz})$ with 100 stirrer positions and the 3 antenna polarizations.

The AUTs were tested in a cylindrical phantom with 12$\mathrm{cm}$ diameter, 28-cm length and $0.2 \mathrm{~cm}$ PVC wall thickness described in [28]. The phantom enclosure was filled with a lossy dielectric solution that mimics the characteristics of human muscle tissue at the mid band frequency of $2250 \mathrm{MHz}$. The muscle equivalent liquid was originally developed for $2450 \mathrm{MHz}$ in [29] but the glycol based solution was sufficiently well matched to the Gabriel tissue properties [30] with a measured relative permittivity $\varepsilon_{\mathrm{r}}=54.6$ and conductivity $\sigma=1.64 \mathrm{Sm}^{-1}$ at $2250 \mathrm{MHz}$ (target values $\varepsilon_{\mathrm{r}}=53.0$ and $\sigma=1.61 \mathrm{Sm}^{-1}$ [30]). A muscle equivalent liquid was chosen as it is one of the major tissue types in the human body and its high signal attenuation demonstrates the sensitivity of the reverberation chamber method. In all cases the AUT was held by a $30-\mathrm{cm}$ rigid coaxial cable (RG405) and connected to the VNA by a $0.91-\mathrm{m}$ Gore Phaseflex cable using an adjustable stand. All cables including the feed cable holding the AUT inside the liquid were calibrated using an automatic calibration unit and were included in the RC reference measurement. The AUT was positioned within the muscle equivalent liquid phantom as illustrated in Fig. 1. The long cylindrical shape of the phantom ensures that the dominating wave propagation from the antenna-phantom system is radial and the cable radiation effects can be readily investigated by changing the level of the muscle-equivalent liquid and the vertical position of the AUT within the cylinder. In Fig. 1, the liquid level from the base of the phantom is denoted as $l$ and $h$ is the AUT's feed point position from the base of the phantom. The feed cable and the main axis of the AUT were always congruent with the cylindrical axis of the phantom.

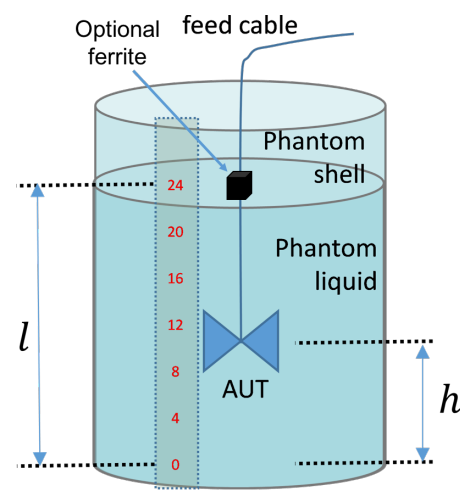

Fig. 1. Schematic of experimental set-up showing AUT positioning with feedpoint at height $h$ within the liquid phantom which has a total height of $l$.

TABLE I

AUT DIMENSIONS

\begin{tabular}{ccccc}
\hline Antenna Type & Monopole & SD & PFMD & SLM \\
\hline $\begin{array}{c}\text { Dimensions } \\
(\mathbf{m m})\end{array}$ & $\begin{array}{c}\text { Radius: } 10 \\
\text { Length: } 5\end{array}$ & \multirow{2}{*}{ Arm: 5 } & $\begin{array}{c}\text { Length: 16 } \\
\text { Width: 16 }\end{array}$ & $\begin{array}{c}\text { Length: } 30 \\
\text { Width: 25 }\end{array}$ \\
\hline
\end{tabular}

A diversity of antennas with different radiation characteristics were utilized in this work to investigate cable radiation effects and to ensure the broad applicability. Two insulated and two un-insulated antenna types were used. Implant antennas are usually insulated as this helps in maintaining the same feed point impedance in proximity to different tissue types [31] and the insulation can be achieved using biocompatible materials. Empirical testing was conducted in [5] to study the coating effect of implant antenna insulation. In their analysis, they compared measurements and simulations of path loss and input impedance and found that using an insulating layer helps to improve overall performance.

The insulated AUTs were a Printed Folded Meandered Dipole (PFMD) and a Slot Loaded Monopole (SLM) as presented in [32]. The PFMD is an inherently robust tissueindependent design with relatively small volume. Two uninsulated canonical AUTs, a sleeve dipole (SD) and monopole, were also used to study closely coupled cases and the effect of the monopole ground plane on cable radiation. All AUTs were matched in the muscle tissue equivalent liquid. The AUTs are shown in Fig. 2 with their dimensions given in Table I. Fig. 3 shows the reflection coefficient $\left(\left|S_{11}\right|\right)$ for each AUT positioned at the center of the full tissue equivalent phantom ( $l=24 \mathrm{~cm}, h=12 \mathrm{~cm})$ as recorded over an extended measurement bandwidth $(2.2-2.6 \mathrm{GHz})$ within the RC. From Fig. 3 it can be seen that all antennas are best matched in the shaded frequency range $(2.2-2.3 \mathrm{GHz})$. It should be noted that for un-insulated AUTs, the tight coupling between the antenna and the lossy material environment led to relatively poorer impedance matching.

\section{RADiATION EFFICIENCY MEASUREMENTS}

Since the objective of this work is to measure the low antenna efficiencies associated with deep implant applications it was important to establish the measurement noise floor. The AUT was replaced with a female SMA $50-\Omega$ load and positioned at the end of the feed cable at the center of the full tissue equivalent phantom $(l=24 \mathrm{~cm}, h=12 \mathrm{~cm})$. The 


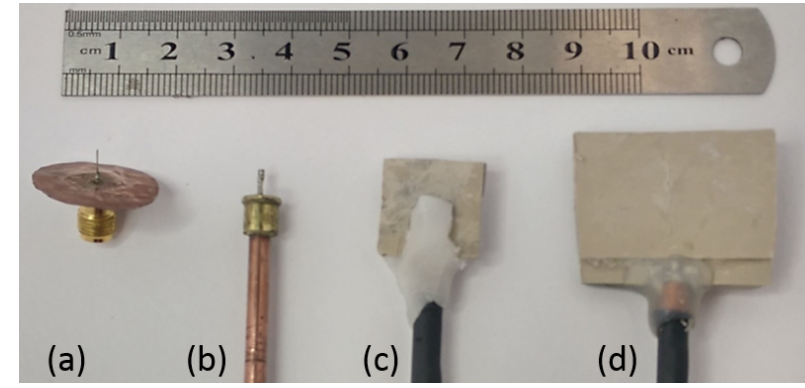

Fig. 2. (a) monopole, (b) SD, (c) PFMD [32], (d) SLM [32].

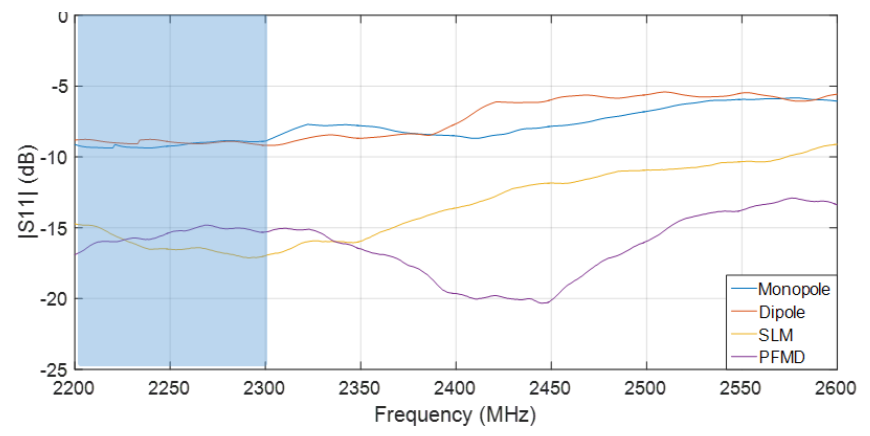

Fig. 3. Measured reflection coefficient $\left(\left|S_{11}\right|\right)$ for all antenna types in muscle equivalent liquid.

recorded total radiation efficiency (which includes the effect of any mismatch) was found to be $-42 \mathrm{~dB}$ on average over the $2.2-2.3 \mathrm{GHz}$ band which is more than $10 \mathrm{~dB}$ below the lowest antenna efficiency results presented in this paper. This provides significant confidence in the results presented and also indicates the lower bound of efficiency measurement for this particular experimental setup.

Three experimental scenarios were selected to highlight the effect of the radiation characteristics of the AUT and feed cable arrangements. The scenarios are categorized based on the combinations of AUT position inside the muscle liquid and liquid level inside the cylindrical phantom. As the phantom is homogeneous, a major factor affecting radiation efficiency is the minimum distance from the AUT to the outside of the phantom. Each scenario was conducted twice to assess its reproducibility and the measurements were conducted in an air conditioned laboratory at a constant temperature to ensure consistent phantom dielectric properties.

\section{A. Scenario 1: full phantom with variable antenna position}

In Scenario 1 the phantom was fully filled with tissue equivalent liquid $(l=24 \mathrm{~cm})$ and the AUT's vertical position (h) was adjusted at intervals of $2 \mathrm{~cm}$ from $8-\mathrm{cm}$ to $22-\mathrm{cm}$ to ensure the antenna structure is fully immersed inside the muscle liquid and at the same time keeping the radial distance the shortest path to the outside boundary of the phantom. The results radiation results are shown in Fig. 4. They illustrate how all antenna types have higher radiation efficiency when the proportion of feed cable within the muscle liquid is shorter, i.e., when $l-h$ is lowest and the antenna is at the top of the phantom. Here the only variable is the length of feed cable that is loaded with lossy muscle equivalent material. This confirms that the cable is radiating. As seen in Fig. 4, in some cases there is a slight $(<1 \mathrm{~dB})$ difference between trials for the same antenna which was due to manual positioning errors. The shaded region refers to the measurements obtained when the radial distance to the outer edge of the phantom is not the shortest distance. For instance, when $h=22 \mathrm{~cm}$, the distance from the AUT's feed point to the top of the phantom $(l)$ is only $2-\mathrm{cm}$, while the radial distance is between 4.5 and $5.8 \mathrm{~cm}$ depending on the AUT. It is worth remarking that the results for the SD are limited to $18-\mathrm{cm}$ (Fig. 4). This was due its construction, in particular it had $6-\mathrm{cm}$ of un-insulated solid coaxial cable which was attached via an SMA connector to the main feed cable. Because of its design, this $6 \mathrm{~cm}$ length was always inserted inside the liquid.

In all cases the minimum radiation efficiency was observed when the AUT was positioned at $h=12 \mathrm{~cm}$. This is the point where the maximum volume of liquid surrounds the antenna in all directions. Likewise, for all positions where $h \leq 18 \mathrm{~cm}$, the trend is the same for all AUTs regardless of radiation characteristics. However, for $h>18 \mathrm{~cm}$ there is a marked difference between the monopole and the PFMD and SLM as monopole efficiency only increases slightly compared to the others due to its inherently radial radiation mode and inclusion of the ground plane. Also, the PFMD and SLM antennas both show significant increase $(>3 \mathrm{~dB})$ as $h$ increases above 18 $\mathrm{cm}$ since the shortest distance to the outside of the phantom is now the top surface of the liquid. The results show that both antenna type and positioning 'depth' within the phantom are critical factors in measuring radiation efficiency.

The PFMD was the most representative of a practical design and even though it has an integral balun [32], the results in Fig. 4 still show considerable cable effect, which diminishes as the tissue equivalent liquid level is increased. Therefore, Scenario 1 was repeated for the PFMD antenna with a split co-axial ferrite core (Wurth Elektronik $100 \Omega, 2.5 \mathrm{GHz}$ ) positioned on the feed cable at the top of the liquid phantom $(l=24 \mathrm{~cm})$ to see if the spurious cable radiation could be further reduced.

On average across two trials with the ferrite core there was a $0.4 \mathrm{~dB}$ lower radiation efficiency for all measurement points compared to the standard case. However, the effect of adding the ferrite was inconsistent, with some AUT positions producing higher efficiency values. Furthermore, the results still exhibited the same trend as seen in the non-ferrite Scenario 1 results for the PFMD shown in Fig. 4.

\section{B. Scenario 2: fixed antenna with variable phantom height}

In this case, the AUT position was fixed with the feed point $6-\mathrm{cm}$ from the base of the phantom $(h=6 \mathrm{~cm})$ and central to the vertical axis of the cylinder. The liquid level $(l)$ was increased from $8-\mathrm{cm}$ (minimal phantom) to $24-\mathrm{cm}$ (full phantom) in 2-cm steps. The efficiency was then measured at each liquid level without changing the AUT position. When the liquid level $(l)$ increased beyond 12-cm, the shortest path from the antenna to the outside of the phantom is always the radial distance. Consequently, any changes in the measured antenna efficiency are due to cable radiation effects or bulk absorption because of the greater volume of lossy material in the phantom. The results are shown in Fig. 5 and there is generally better agreement between the experimental trials. The shaded region 


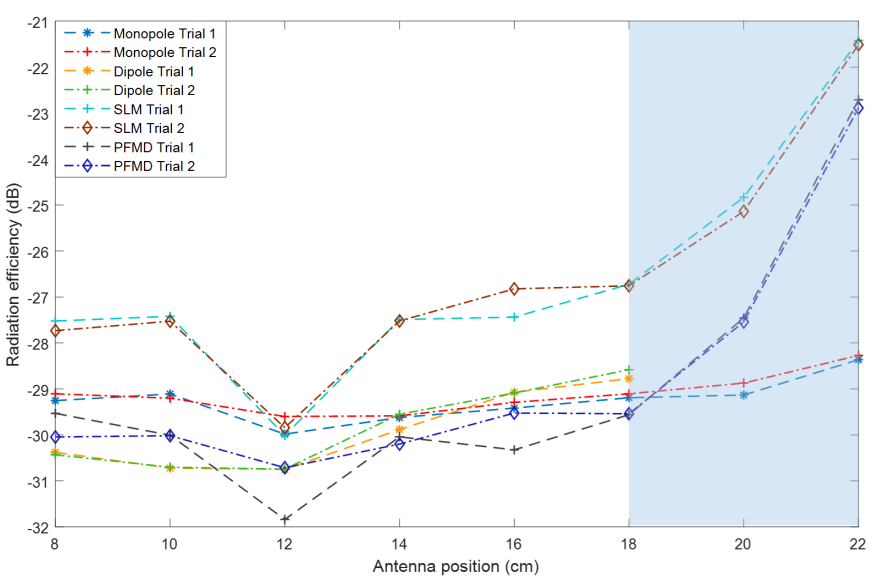

Fig. 4. Total radiation efficiency with fixed liquid level at $24-\mathrm{cm}$ and variable AUT position. Shaded region is when distance from antenna feed point to the top surface of the liquid phantom $<$ the radial distance $(l-h<4.5 \mathrm{~cm})$.

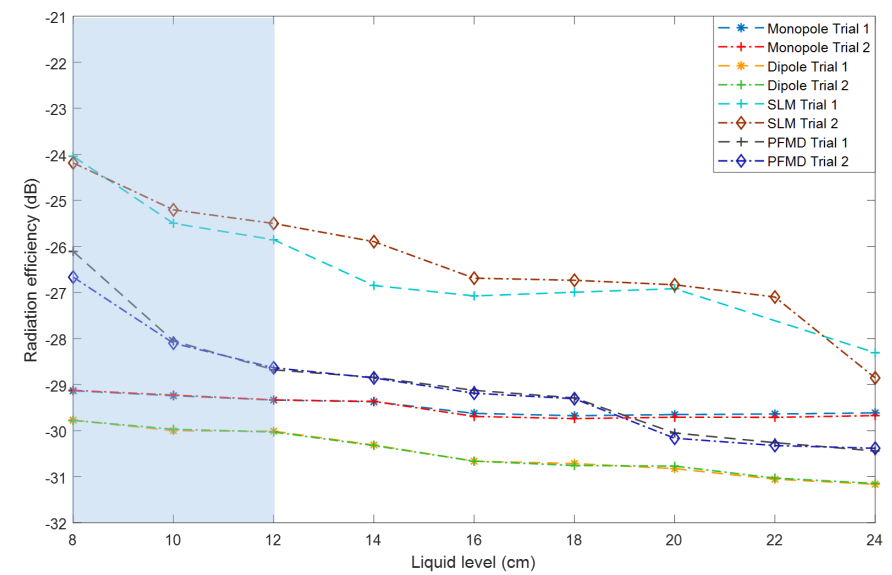

Fig. 5. Total radiation efficiency with AUT at $6 \mathrm{~cm}$ and variable liquid level. Shaded region is when the distance from the antenna feed point to the top surface of the liquid phantom $<$ radial distance $(l-6<4.5 \mathrm{~cm})$.

indicates when the distance from the AUT feed point to the liquid level is shorter than the radial distance. Although the overall radiation efficiency differs from one antenna to the other, the measurements show how any increase in liquid level reduces the radiation efficiency for all antennas. However, since both the phantom volume and feed cable length within the phantom $(l-h)$ are varying in this scenario it is not possible to differentiate between these effects.

This scenario also highlights the variation in radiation performance between the AUTs. Notably, the monopole is the least affected by cable and phantom material absorption effects as it has the lowest change in radiation efficiency as the phantom liquid height is increased (Fig. 5) with less than $1 \mathrm{~dB}$ difference between the $l=12 \mathrm{~cm}$ case and the full phantom $(l=24 \mathrm{~cm})$. The difference between this scenario and Scenario 1 is that the phantom volume on the ground plane side of the monopole antenna is increased but the extra loading has virtually no effect with the change in monopole efficiency being of a similar scale across both Fig. 4 and Fig. 5. The results in Fig. 5 also show that while the SLM and PFMD have the highest efficiency values in general, the scale of this advantage reduces as the phantom volume increases which must be due to a combination of dampening of feed cable radiation and bulk tissue material absorption effects.

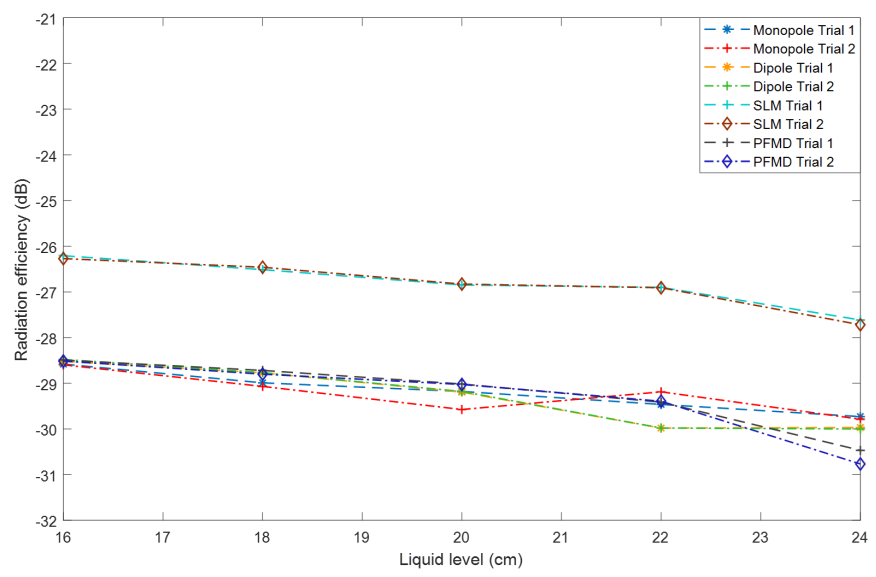

Fig. 6. Total radiation efficiency with AUT fixed at centre of variable liquid level phantom.

\section{Scenario 3: antenna fixed in center of variable phantom}

In Scenario 3 the phantom level was varied (similar to Scenario 2) but in this case the vertical position of the AUT was set to the mid-point of the liquid height $(h=l / 2)$. Contrasted with Scenario 2, this helps to identify the bulk absorption effect of a larger phantom volume, although it is also subject to cable effects. The liquid level $(l)$ was increased from $16-\mathrm{cm}$ to $24-\mathrm{cm}$ (full phantom) in $2 \mathrm{~cm}$ steps and the AUT's vertical position $(h)$ was then simultaneously adjusted at intervals of $1 \mathrm{~cm}$ each from $8-\mathrm{cm}$ to $12-\mathrm{cm}$. Note that in this scenario the shortest path from the antenna to the outside of the phantom is always the radial distance. The results shown in Fig. 6 demonstrate how the AUT efficiency reduces as the phantom size increases. The results are consistent in terms of the difference between antenna types with the physically largest antenna (SLM) being consistently $2 \mathrm{~dB}$ more efficient.

\section{Cable Effect Analysis and Validation}

The results in Section IV show that, regardless of antenna type, the low radiation efficiencies associated with deep tissue implant antennas mean that RF cable based measurement effects can directly affect the results obtained. To analyse this further, the results were collated for all cases where the antenna was positioned such that the shortest distance for radiation is the radial distance (i.e., cable length inside liquid $>5 \mathrm{~cm}$ ) and the total radiation efficiency plotted in Fig. 7. The results are the average efficiency for each AUT for each cable length within the phantom across all scenarios. The average is used as there are a number of cases in the three scenarios which have the same cable length inside the phantom but the overall phantom volume may be different. The average allows us to focus solely on the cable effect. This graphical analysis highlights the main conclusions of this study as it demonstrates how the antenna efficiency values are dependent on its position with respect to the outer boundaries of the phantom. The plot in Fig. 7 also shows the logarithmic trend line associated with each AUT with the corresponding attenuation rates shown in Table II. The trend lines show that, irrespective of antenna type and overall phantom volume, the measured radiation efficiency decreases as the length of cable within the liquid phantom increases. The surrounding lossy tissue-equivalent material is dampening the radiation field 


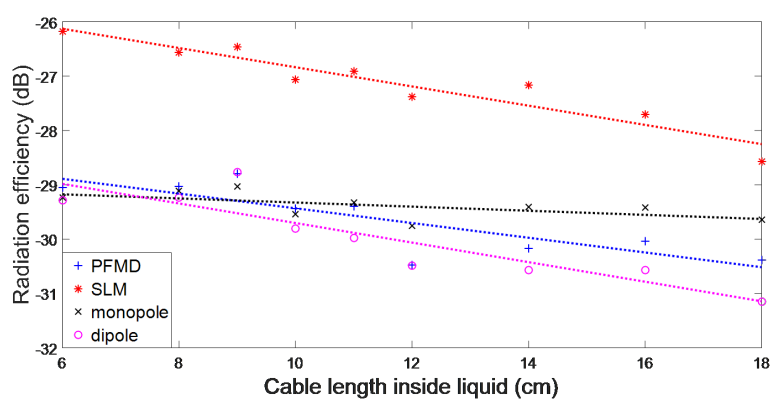

Fig. 7. Average radiation efficiency results for all cases where the cable length inside the liquid tissue phantom is at least $5 \mathrm{~cm}$.

associated with the feed cable. However, the magnitude of this cable radiation effect significantly differs from one antenna to another according to the coupling to the feed cable. The uninsulated monopole has a significantly lower attenuation rate due to its ground plane which reduces the currents flowing on the outside of the feed cable, reducing spurious radiation. The insulated SLM and the un-insulated SD are most affected by the feed cable arrangements. This effect is less apparent in the case of the PFMD which is most likely due to the integrated balun that is incorporated in that design [32].

It is important to consider the precision and repeatability of the radiation efficiency results as, given the high attenuation of phantom material, small errors in antenna positioning ('depth' within the phantom) can be a significant factor. To investigate this, the results in Section IV were examined to identify 'matched' cases where phantom liquid level and antenna position were identical. Given that the experiments for each scenario were conducted at different times, it is equivalent to having additional trials for all of the corresponding configurations. These matching cases occurred with Scenario $1(h=12$ $\mathrm{cm})$ and Scenario $3(l=24 \mathrm{~cm})$; and Scenario $1(h=8 \mathrm{~cm})$ and Scenario $2(l=24 \mathrm{~cm})$. For each antenna type the largest difference between these matched cases is shown in Table II. Overall, the difference between matched cases is always less than $1.1 \mathrm{~dB}$ and this is comparable with the positioning error found during the two trials performed for each scenario.

To estimate the maximum error caused by cable radiation we calculated the range of efficiency results across both Scenarios 1 (Fig. 4) and 2 (Fig. 5) for all cases where the antenna was positioned such that the shortest distance for radiation is the radial distance (i.e., cable length inside liquid $>5 \mathrm{~cm}$ ) and the results are shown in Table II. Again, due to its radiation characteristics, the monopole was less susceptible to cable errors, whereas the SLM error was over $3 \mathrm{~dB}$ higher.

To validate our empirical results, Finite-Difference TimeDomain simulations were conducted using SEMCAD $\mathrm{X}$ (Schmid \& Partner Eng. AG, Zurich, Switzerland). The PFMD and SLM antennas were simulated in a numerical muscle tissue phantom with a discrete $2250 \mathrm{MHz}$ source to estimate $\eta$ in the absence of feed cable effects. The muscle tissue properties were identical to the measured phantom liquid with $\varepsilon_{\mathrm{r}}=54.6$ and $\sigma=1.64 \mathrm{Sm}^{-1}$. The radius of the phantom was held constant but the simulations were repeated with different cylinder lengths between $16-24 \mathrm{~cm}$ and various antenna positions to represent the three measurement scenarios. The simulated total radiation efficiency remained effectively constant irrespective
TABLE II

SUMMARY OF RESULTS

\begin{tabular}{ccccc}
\hline Antenna Type & Monopole & SD & PFMD & SLM \\
\hline Attenuation Rate $(\mathbf{d B} / \mathbf{c m})$ & 0.04 & 0.18 & 0.14 & 0.18 \\
Max. matched case error $(\mathbf{d B})$ & 0.3 & 1.1 & 1.0 & 0.9 \\
Max. cable error $(\mathbf{d B})$ & 0.9 & 2.6 & 3.2 & 4.5 \\
\hline
\end{tabular}

of phantom volume and antenna positioning: an average of $-28.3 \mathrm{~dB}$ for the SLM and $-32.5 \mathrm{~dB}$ for the PFMD across all three scenarios, with a maximum variation of $0.4 \mathrm{~dB}$ and $0.1 \mathrm{~dB}$, respectively. There was good agreement with the measured results. For example, for the Scenario 1 mid point $(12-\mathrm{cm})$ case, the simulated SLM efficiency was $-28.6 \mathrm{~dB}$ and the PFMD was $-33.1 \mathrm{~dB}$, both within $1.3 \mathrm{~dB}$ of the results in Fig. 4. This provides confidence in the proposed method, taking into account unavoidable positioning and systematic measurement system errors.

\section{CONClusion AND ReCOMmEndations}

Radiation efficiency is an important metric for implant antennas as it directly affects application link budgets. While propagation path losses in body tissues are unavoidable, losses close to the radiator are heavily dependent on the antenna, its packaging and radiation characteristics. We have shown the accurate and repeatable measurement of deep implant antenna radiation efficiency using a reverberation chamber method. By considering a range of antennas and a variety of measurement scenarios, it is demonstrated that the RC method can accurately measure implant antenna efficiencies as low as $0.06 \%(-32 \mathrm{~dB})$ with a repeatability, even for manual positioning, of around $1 \mathrm{~dB}$ within an $\mathrm{RC}$ with a known efficiency measurement accuracy of $0.5 \mathrm{~dB}$. More accurate results would require robotic positioning and better chamber and measurement system specifications. The different scenarios considered highlighted the importance of the AUT's position in any phantom. Care must be taken to ensure that the main propagating path (most likely shortest distance to the surface) is representative of the application and not an artefact of the measurement set up. Likewise, the change in bulk absorption due to the overall phantom volume may be a factor, although this depends on both radiation characteristics and the dominance of the shortest path in a particular phantom.

One of the main outcomes is the characterization of spurious radiation from feed cables and how this is strongly dependent on the AUT radiation characteristics. The results showed strong cable effect except where the cable's radiation was dampened by the lossy material of the phantom. Ensuring that the feed cable is loaded by an appropriate depth of phantom material was sufficient to see a significant reduction (0.9$4.5 \mathrm{~dB}$ for the antennas studied) in spurious radiation. Given the low efficiencies of deep implant antennas, it is also recommended that the noise floor is measured to ensure sensitivity for the particular AUT and phantom utilized. Radiation efficiency measurements are difficult and often avoided in implant antenna studies, whereas numerical simulations are regularly reported. Yet it remains important to be able to empirically validate results and the RC approach is practical, repeatable and, subject to the specifications of the measurement system, sensitive enough for deep tissue implant antennas. 


\section{REFERENCES}

[1] K. Gulati, F. Yazicioglu, A. Dupret, R. Genov, P. C.-Y. Wu, and L. Yan, "F6: Circuit, systems and data processing for next-generation wearable and implantable medical devices," in 2016 IEEE International SolidState Circuits Conference (ISSCC), Jan 2016, pp. 510-513.

[2] S. K. Islam, A. Fathy, Y. Wang, M. Kuhn, and M. Mahfouz, "HassleFree Vitals: BioWireleSS for a Patient-Centric Health-Care Paradigm," IEEE Microwave Magazine, vol. 15, no. 7, pp. S25-S33, Nov 2014.

[3] M. Hanson, H. Powell, A. Barth, K. Ringgenberg, B. Calhoun, J. Aylor, and J. Lach, "Body Area Sensor Networks: Challenges and Opportunities," Computer, vol. 42, no. 1, pp. 58-65, Jan 2009.

[4] A. Khaleghi, I. Balasingham, and R. Chaavez-Santiago, "Computational study of ultra-wideband wave propagation into the human chest," IET Microwaves, Antennas \& Propagation, vol. 5, no. 5, pp. 559-567, 2011.

[5] S.-M. Huang, M.-R. Tofighi, and A. Rosen, "Considerations for the Design and Placement of Implantable Annular Slot Antennas for Intracranial Pressure Monitoring Devices," IEEE Antennas and Wireless Propagation Letters, vol. 14, pp. 1514-1517, 2015.

[6] H. Yoo and Y. Cho, "Miniaturised dual-band implantable antenna for wireless biotelemetry," Electronics Letters, vol. 52, no. 12, pp. 10051007, Jun 2016

[7] R. Chavez-Santiago, C. Garcia-Pardo, A. Fornes-Leal, A. Valles-Lluch, G. Vermeeren, W. Joseph, I. Balasingham, and N. Cardona, "Experimental Path Loss Models for In-Body Communications within 2.36-2.5 GHz," IEEE Journal of Biomedical and Health Informatics, vol. 19, no. 3, pp. 1-1, 2015.

[8] L. Schmitt, J. Espina, T. Falck, and D. Wang, "Biosensor Communication Technology and Standards," in Handbook of Biomedical Telemetry. Hoboken, NJ, USA: John Wiley \& Sons, Inc., Aug 2014, pp. 330-367.

[9] Li-Jie Xu, Yong-Xin Guo, and Wen Wu, "Miniaturized Dual-Band Antenna for Implantable Wireless Communications," IEEE Antennas and Wireless Propagation Letters, vol. 13, pp. 1160-1163, 2014.

[10] A. Kiourti and K. S. Nikita, "A Review of Implantable Patch Antennas for Biomedical Telemetry: Challenges and Solutions [Wireless Corner]," IEEE Antennas and Propagation Magazine, vol. 54, no. 3, pp. 210-228, Jun 2012.

[11] X. Meng, K. D. Browne, S.-M. Huang, C. Mietus, D. K. Cullen, M.R. Tofighi, and A. Rosen, "Dynamic Evaluation of a Digital Wireless Intracranial Pressure Sensor for the Assessment of Traumatic Brain Injury in a Swine Model," IEEE Transactions on Microwave Theory and Techniques, vol. 61, no. 1, pp. 316-325, Jan 2013.

[12] W. G. Scanlon, J. B. Burns, and N. E. Evans, "Radiowave propagation from a tissue-implanted source at $418 \mathrm{MHz}$ and 916.5 MHz." IEEE transactions on bio-medical engineering, vol. 47 , no. 4, pp. 527-34, Apr 2000.

[13] C. L. Holloway, H. A. Shah, R. J. Pirkl, W. F. Young, D. A. Hill, and J. Ladbury, "Reverberation Chamber Techniques for Determining the Radiation and Total Efficiency of Antennas," IEEE Transactions on Antennas and Propagation, vol. 60, no. 4, pp. 1758-1770, Apr 2012.

[14] C. L. Holloway, R. S. Smith, C. R. Dunlap, R. J. Pirkl, J. Ladbury, W. F. Young, D. A. Hill, W. R. Hansell, M. A. Shadish, and K. B. Sullivan, "Validation of a two-antenna reverberation-chamber technique for estimating the total and radiation efficiency of antennas," in International Symposium on Electromagnetic Compatibility - EMC EUROPE, Sept 2012, pp. 1-6.

[15] G. Conway, W. Scanlon, C. Orlenius, and C. Walker, "In Situ Measurement of UHF Wearable Antenna Radiation Efficiency Using a Reverberation Chamber," IEEE Antennas and Wireless Propagation Letters, vol. 7, pp. 271-274, 2008.

[16] M. Moharram and A. Kishk, "MIMO Antennas Efficiency Measurement Using Wheeler Caps," IEEE Transactions on Antennas and Propagation, vol. 64, no. 3, pp. 1115-1120, Mar 2016.

[17] G. L. Fur, C. Lemoine, P. Besnier, and A. Sharaiha, "Comparison of efficiency measurements for narrow band antennas using UWB Wheeler Cap and Reverberation Chamber," in 2009 3rd European Conference on Antennas and Propagation, Mar 2009, pp. 2682-2686.

[18] A. Khaleghi, "Time-Domain Measurement of Antenna Efficiency in Reverberation Chamber," IEEE Transactions on Antennas and Propagation, vol. 57, no. 3, pp. 817-821, Mar 2009.

[19] C. Sánchez-Fernández, O. Quevedo-Teruel, J. Requena-Carrión, L. Inclán-Sánchez, and E. Rajo-Iglesias, "Dual-band microstrip patch antenna based on short-circuited ring and spiral resonators for implantable medical devices," IET Microwaves, Antennas \& Propagation, vol. 4, no. 8, pp. 1048-1055, 2010.
[20] K. Ito, "Human body phantoms for evaluation of wearable and implantable antennas," in The Second European Conference on Antennas and Propagation, EuCAP 2007, Nov 2007, pp. 1-6.

[21] F. Merli and A. K. Skrivervik, "Design and measurement considerations for implantable antennas for telemetry applications," in Proceedings of the Fourth European Conference on Antennas and Propagation. EurAAP, 2010, pp. 1-5.

[22] A. Skrivervik, J.-F. Zurcher, O. Staub, and J. Mosig, "PCS antenna design: the challenge of miniaturization," IEEE Antennas and Propagation Magazine, vol. 43, no. 4, pp. 12-27, 2001.

[23] A. Scannavini, L. J. Foged, and L. Scialacqua, "Testing electrically small antennas with using a fiber optic to RF transducer," in 2014 IEEE Antennas and Propagation Society International Symposium (APSURSI), July 2014, pp. 271-272.

[24] T. H. Loh, "Non-invasive measurement of electrically small ultrawideband and smart antennas," in 2013 Loughborough Antennas Propagation Conference (LAPC), Nov 2013, pp. 456-460.

[25] M. Moharram and A. Kishk, "Contactless Wheeler cap concept for antenna radiation efficiency measurements," in 2014 XXXIth URSI General Assembly and Scientific Symposium (URSI GASS). IEEE, Aug 2014, pp. 1-4.

[26] R. Vogt-Ardatjew, A. E. Sowa, and F. Leferink, "The effect of magnetodielectric absorbing coating on unsymmetrical antenna cables," in 2015 Asia-Pacific Symposium on Electromagnetic Compatibility (APEMC). IEEE, May 2015, pp. 624-627.

[27] S. S. Zhekov, A. Tatomirescu, and G. F. Pedersen, "Modified biconical antenna for ultrawideband applications," in 2016 10th European Conference on Antennas and Propagation (EuCAP), April 2016, pp. 1-5.

[28] P.-S. Kildal and C. Carlsson, "TCP of 20 Mobile Phones Measured in Reverberation Chamber: Procedure, Results, Uncertainty and Validation available from, Bluetest AB," Bluetest AB [Online], 2002. [Online]. Available: https://bluetest.se/

[29] G. A. Conway and W. G. Scanlon, "Antennas for Over-Body-Surface Communication at $2.45 \mathrm{GHz}$," IEEE Transactions on Antennas and Propagation, vol. 57, no. 4, pp. 844-855, Apr 2009.

[30] S. Gabriel, R. Lau, and C. Gabriel, "The dielectric properties of biological tissues: II. Measurements in the frequency range $10 \mathrm{~Hz}$ to $20 \mathrm{GHz}, "$ Physics in Medicine and Biology, vol. 41, no. 11, pp. 2251$2269,1996$.

[31] J. Gemio, J. Parron, and J. Soler, "Human Body effects on Implantable Antennas for ISM Bands Applications: Models Comparison and Propagation Losses Sudy," Progress In Electromagnetics Research, vol. 110, pp. 437-452, 2010.

[32] M. K. Magill, G. A. Conway, and W. G. Scanlon, "Tissue-Independent Implantable Antenna for In-Body Communications at $2.36-2.5 \mathrm{GHz}$," IEEE Transactions on Antennas and Propagation, vol. 65, no. 9, pp. 4406-4417, Sept 2017.

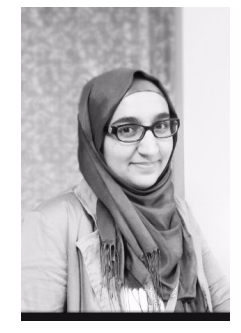

Yomna El-Saboni received her B.Sc. degree in electrical engineering with a minor in biomedical engineering from the American University of Sharjah, UAE in 2013 and her M.Sc. in medical engineering from the University of Leeds, UK in 2014. She is currently working on her Ph.D. degree in Electronic Engineering in the area of medical implant wireless body-centric communications and sensors. Her research interests include miniaturized medical implantable devices and robotics, electrically small implantable antennas, computational electromagnetism for biomedical applications and wireless communication between several implantable devices within the human body. 


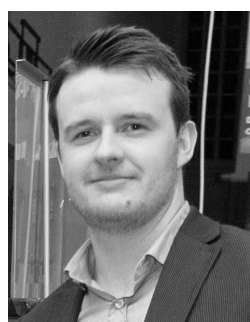

Matthew K. Magill received a Masters of Engineering degree in Electrical and Electronic Engineering (with first class honors) from the Queen's University of Belfast in 2014 where he is currently working on a $\mathrm{Ph} . \mathrm{D}$. degree in Electronic Engineering in the area of medical wireless body-centric communications and sensors. His research interests include implantable and wearable antenna design, computational electromagnetism, human tissue equivalent materials, implantable system test-beds and implantable experimental test methodologies for in-body communica-

tions.

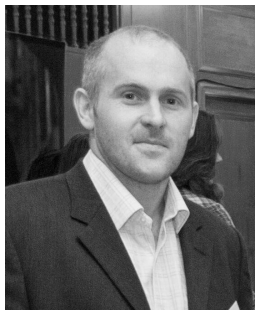

Gareth A. Conway received a BEng Hons. degree in Electronic Systems from Ulster University, UK in 2004. In 2008, he completed a Ph.D. degree in Electronic Engineering, entitled 'Wearable Antennas for On-Body Wireless Communications' at Queen's University of Belfast, (UK). On completion of his doctorate, he spent three years as a commercial research engineer, specializing in antennas and propagation for mobile communication. In 2011, he rejoined QUB to complete an EPSRC Knowledge Transfer Secondment with Toumaz Healthcare Ltd., undertaking research and development in 'Innovative body-worn antennas for medical devices.' In 2013 he became a Lecturer in Communications Engineering at ECIT, Queens University of Belfast. Dr. Conway has authored or co-authored 35 international conference and journal papers. His research interests include antennas, human tissue equivalent materials, wave propagation and computational electromagnetism for wearable and implantable communications.

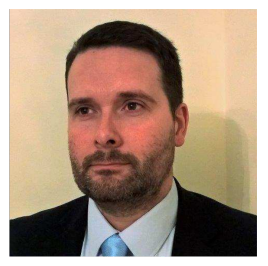

Simon L. Cotton (S'04-M'07-SM'14) received the B.Eng. degree in electronics and software from Ulster University, U.K., in 2004, and the Ph.D. degree in electrical and electronic engineering from the Queen's University of Belfast, Belfast, U.K., in 2007. He is currently a Reader of Wireless Communications with the Institute of Electronics, Communications and Information Technology, Queen's University Belfast, and also a Co-Founder and the Chief Technology Officer with ActivWireless Ltd., Belfast. He has authored and co-authored over 100 publications in major IEEE/IET journals and refereed international conferences, two book chapters, and two patents. Among his research interests are cellular device-to-device, vehicular, and body-centric communications. His other research interests include radio channel characterization and modeling and the simulation of wireless channels. He was a recipient of the $\mathrm{H}$. A Wheeler Prize, in 2010, from the IEEE Antennas and Propagation Society for the best applications journal paper in the IEEE TRANSACTIONS ON ANTENNAS AND PROPAGATION in 2009. In 2011, he was a recipient of the Sir George Macfarlane Award from the U.K. Royal Academy of Engineering in recognition of his technical and scientific attainment since graduating from his first degree in engineering.

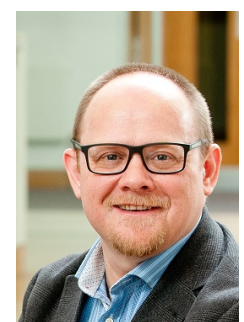

William G. Scanlon (M'98-SM'13) received the $\mathrm{Ph} . \mathrm{D}$. degree in electronics, specializing in wearable and implanted antennas, from Ulster University, UK in 1997. He was appointed as Lecturer at the University of Ulster in 1998, Senior Lecturer and Full Professor at Queen's University of Belfast (UK) in 2002 and 2008, respectively. He is currently Chair of Wireless Communications and Director of the Centre for Wireless Innovation at Queen's and he held a part-time Chair in Short Range Radio at the University of Twente, The Netherlands from 2009 to 2014. He is a pioneer in wearable and medical device communications, particularly in relation to implantable solutions, having worked and published in this area since 1995. His other research interests include mobile wireless communications, RF and microwave propagation, channel modelling and characterization, wireless networking and protocols and wireless networked control systems. He served as keynote speaker for the IEEE Intl. Microwave Workshop Series on RF and Wireless Technologies for Biomedical and Healthcare Applications (2014), the NATO Military Communications and Information Systems Conf. (2010), the Intl. Conf. on Bodynets (2010) and the European Workshop on Conformal Antennas (2007). He has been a Series Editor of the IET Book Series on Telecommunications and Networking, he was an inaugural Associate Editor of the IEEE Journal of Translational Engineering in Health and Medicine and he is an Associate Editor for IEEE Antennas and Wireless Propagation Letters. Prof. Scanlon received a Young Scientist award from URSI in 1999, he was a recipient of the 2010 IEEE H. A. Wheeler Prize Paper Award for IEEE Trans. Antennas and Propagation and he delivered the 2012 NATO International Lecture Series on Next Generation Communications. 